\title{
Applications of a Compact Ultrasonic 3D Sensor for Recognition of Shape and Orientation of Objects
}

\author{
Christian Walter ${ }^{1}$, Herbert Schweinzer ${ }^{2}$ \\ ${ }^{1}$ Vienna University of Technology, E354, Gußhausstrasse 25/354, A-1040 Vienna \\ cwalter@emce.tuwien.ac.at \\ ${ }^{2}$ Vienna University of Technology, E354, Gußhausstrasse 25/354, A-1040 Vienna
}

\begin{abstract}
Ultrasonic sensors are well established in various industrial, medical and commercial applications. A specific subclass of these applications employs ultrasonic sensors to obtain information of objects within the vicinity of the sensor. Using a compact 3D-ultrasonic sensor as a basic building block, applications can be classified into three different groups, based on the reference system used. The first group employs a global reference system, in which position and orientation of the sensor are known, and whereas the objects are described herein. Possible applications include scene analysis where the sensor detects objects within the environment. The second group uses the reference system of the sensor where the sensor is not expected to move after initial calibration. Multiple fixed sensors can be used for identifying and localizing of possibly moving objects. The third application uses the sensor in combination with a movable robot controlled by sensor information. The sensor can be seen similar to the navigation system of a bat. Two example applications will be highlighted within this article showing the applicability of the system using a global reference system for scene analysis, and secondly employing two compact 3D sensors operating in a group, to identify size and orientation of primitive objects on a linear belt.
\end{abstract}

Key words: ultrasonic sensor, object recognition, object classification, scene analysis, robotics

\begin{abstract}
Introduction
Ultrasonic sensors are well established in various industrial, medical and commercial applications. Maybe the most important difference compared to optical systems is, that in typical industrial environments data obtained by ultrasonic sensors is due to specular reflection, whereas optical devices deliver images by diffuse reflection. Furthermore, due to the low propagation speed of $343 \mathrm{~m} / \mathrm{s}$, high resolution distance measurements are easily performed using ultrasonic sensors. Thus measuring ultrasound reflection signals has completely different quality, delivers high accuracy, and is especially suitable for determining parameters of partially defined objects.
\end{abstract}

Determination of parameters is a work which was pioneered by Kuc and Kleeman [1] already 20 years ago. Over the last two decades multiple systems have evolved. Two more recent systems are examples of typical application-oriented systems and shall be briefly introduced. The first system allows the determination of the parameters of three planes using a scanning sensor and principal component analysis (PCA) [2]. For this task two of the shelf sensors are mounted on a tripod.
The total measurement time required is 226 seconds. The developed algorithms and the proposed sensor system work reasonably well and are especially tailored for this specific problem. The second system presented in [3] is a compact 3D-sensor and consists of four transducers, all of them working as sender and receiver, yielding 16 time-of-flight (ToF) measurements in total. Using an initial learning phase, specific features of planes, edges and corners are learned. During measurement the obtained ToF vector is compared to the previously learned data using dimensionality reduction by PCA.

This article proposes a 3D compact ultrasonic sensor which is able to measure the positions of 3D-reflection points produced by specular reflections on object surfaces with low uncertainty, high robustness and limited object dependence at sufficient distance. The proposed sensor construction has evolved from over a decade of research in the area of ultrasonic sensors at the measurement and control system group. One or several of these sensors can be combined to set up a measurement system adjusted to measure parameters of specific type of objects. 
Firstly, this paper will present the concept of the sensor system. Secondly, possible application types are discussed, followed by short presentations of two application scenarios together with realized examples of real measurements. The paper is closed by a discussion of the method and a conclusion.

\section{Concept of a 3D-Sensor based on Ultrasound}

The basic sensor construction is shown in Fig. 1 and consists of an electrostatic transmitter $\mathrm{T}$ and four (or five) microphones M1-M5 mounted $\mathrm{d}=40 \mathrm{~mm}$ apart from the center of the transmitter [4]. The fifth microphone, if used, can be placed arbitrarily. The transmitter is a Senscomp 600 open face electrostatic transducer. The microphones used are Knowles SPM0404UD5 microphones which omni-directional directivity and a sensitivity of $-42 \mathrm{dBV}$ (re $1 \mathrm{~V} / \mathrm{Pa}$ ). The time difference between a microphone pair is bounded by $2 d / c \approx 233 \mu s$, where $c$ is the speed of sound. The peak sensitivity is $4.1 \mu \mathrm{s} /{ }^{\circ}$ in polar/azimuth coordinates where average sensitivity is between $1.3 \mu \mathrm{s} /{ }^{\circ}$ and $2 \mu \mathrm{s} /{ }^{\circ}$ [5]. Therefore high resolution ToF measurements are required and pulse compression techniques are used. The presented sensor uses a linear frequency modulated chirp with a bandwidth $B$ from $35-65 \mathrm{kHz}$ and a duration of $512 \mu \mathrm{s}$.

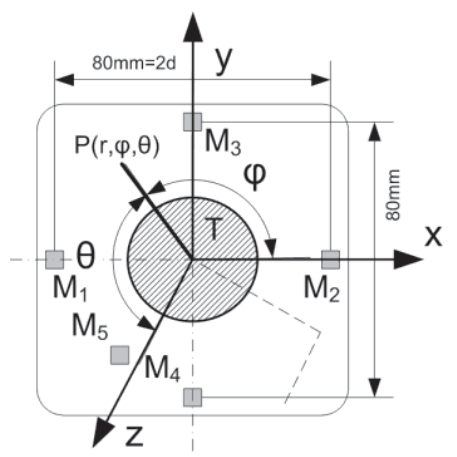

Fig. 1. Compact 3D-sensor with centered transducer and five microphones. The fifth microphone is optional and has no special placement restrictions.

The received signals in each channel are processed using 1-Bit binary correlation where multiple spatially predicted templates are used [6]. The obtained peaks in each channel are used to build groups of time-of-flight (ToF) information using the spatial constraints enforced by the sensor, i.e. the spacing of the microphones. Due to the usage of multiple correlation templates multiple groups might be detected for a single reflector, where a group is considered to be similar, if their respective ToFs are closer than 1/B. To increase robustness of the $3 D$ localization and to select the proper group it is beneficial to employ the methods described in [7]. For each group a criterion is obtained, where the criterion is a combination of the overall correlation quality, the midpoint criterion (defined later) and the consistency with the time-difference-of-arrival between the received signals. The overall correlation quality is a measure how well the signals within each channel match. For binary signals matching can be defined as either all data being 1 or 0 . The midpoint criterion is a geometric feature of the compact 3D sensor and can be used to determine if all reflection points are one the same object, and if the result is consistent at all. If all reflections points are on the same object it can be shown, for arbitrary convex and planar objects at a distance of $>50 \mathrm{~cm}$, that the average of the respective ToF information for the microphone pairs $1 / 2$ and $3 / 4$ is similar by approx. $2 \mathrm{~mm}$. Comparison with TDoA information gives an additional measure, as comparison with this information should give identical results to correlation with the reference patterns. As only a small part of the signal is used this calculation is very fast and can improve reliability as generation of reference patterns assumes an ideal transmitter.

The result of this calculation are groups with high quality ToF information, which can be used to locate objects in 3D using the simple formula shown in (1), where $t_{1}, t_{2}, t_{3}$ and $t_{4}$ are the respective ToF information.

$$
\begin{aligned}
& l_{i}=t_{i} \cdot c r=\frac{l_{1}^{2}+l_{2}^{2}-2 d^{2}}{2\left(l_{1}+l_{2}\right)} \\
& y=r \frac{\left(l_{3}-r\right)^{2}-d^{2}-r^{2}}{-2 d r} \\
& x=r \frac{\left(l_{2}-r\right)^{2}-d^{2}-r^{2}}{-2 d r} \\
& z=\sqrt{r^{2}-x^{2}-y^{2}}
\end{aligned}
$$

\section{Main features of the sensor system}

The proposed compact 3D sensor has the following features making it especially suitable for object recognition in air:

- Does not only determine distance but also directional information of the reflector. Furthermore the direction of the echo equals the surface normal.

- Ability to obtain multiple information of a single measurement due to inherent echo separation capabilities (often also called echo correspondance problem).

- High quality data due to inherent outlier reduction methods. 


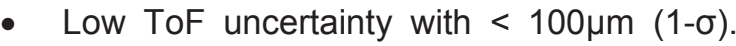
This results in low angular and distance uncertainty, although in Cartesian coordinates uncertainty increases with distance of the sensor.

- Object independent for convex and planar reflectors at distances $>50 \mathrm{~cm}$.

\section{Prerequisites of Setting up a Measurement System for Object Parameters}

Besides of a basic application as a static sensor and static objects, which makes only sense in completely well-defined measurement tasks, movements of sensor or objects is a requirement for getting a sufficient number of data. Three alternatives are feasible:

- Moving sensor with well-defined absolute knowledge of the position of the coordinate system of the sensor in a room coordinate system. In this case, object parameters can be measured with respect to their absolute position in the room. Map building is an example for this type of applications.

- Objects are moved and the sensor system remains on its static position. In this case, object parameters can be identified if the actual object positions are known with respect to any static coordinate system, which can also be the coordinate system of the sensor. A typical example of this case is measuring parameters of objects placed on a conveyor, where actual conveyor position, movement direction and actual speed are known.

- Sensor moving relative to objects with known features respectively parameters. An example of this can be using the sensor mounted on an industrial robot, where the sensor is guiding the robot for reaching precise positions and orientation with respect to a well-known object

\section{Application "Scene Analysis with Moving Sensor"}

Fig. 2 shows an example application where a cylinder with a diameter of $10.5 \mathrm{~cm}$ was placed in front of a cabinet. The aim of this test setup was to automatically identify and classify all objects using multiple measurements at different positions. For this task the sensor was mounted on a linear belt where movement was performed along the $\mathrm{x}$-axis. What is unique in this test setup is that the two doors of the cabinet are not at the same depth. While crossing the intersection of these two doors, the reflection points of the microphones are no longer on the same object, which could result in potentially incorrect data. This situation was used to verify the performance of the echo correspondence and outlier avoidance algorithms. A more detailed description of this problem and the comparison of the results when applying different algorithms is given in [6].

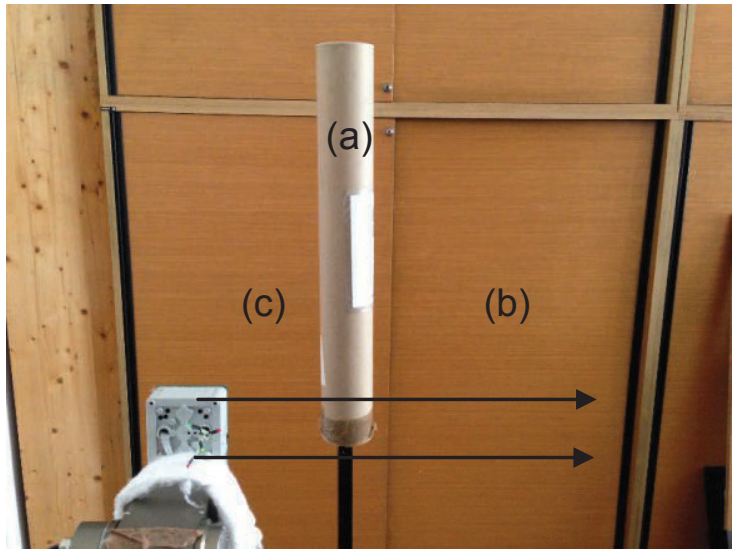

Fig. 2. Example scene consisting of a cylinder (a) and two planar reflectors (b) and (c). The sensor obtains two traces of measurements each covering $50 \mathrm{~cm}$ at different vertical positions.

Eventually a high quality map of 3D reflection points is obtained which is used for scene analysis. The resulting data is shown in Fig. 3 where the reflection points (b), (a) and (c) are due to reflection on the right door, the cylinder and the left door of the cabinet. The objects (b2) and (c2) are "mirror objects" and are due to multiple reflections on the sensor surface. These distances are therefore exactly two times the distance of the objects (b) and (c). Another object labeled (d) is faked due to reflection on the backside surface of the cylinder. The presented data here is shown without any manual post-processing and all algorithms execute fully automatically. Only a very limited number of outliers are present in the final data.

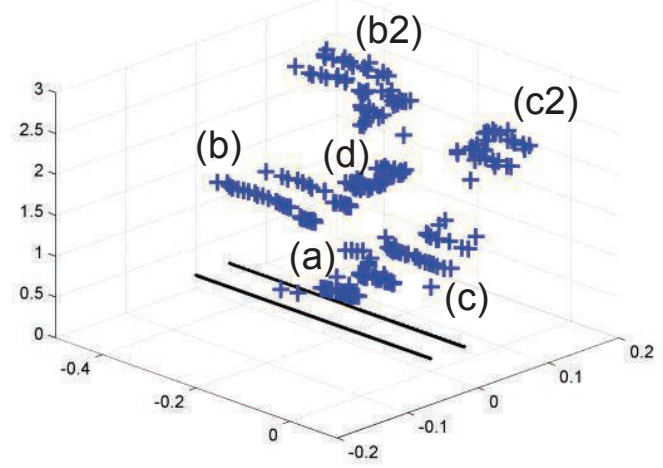

Fig. 3. ToF measurements showing the two planes (b) and (c) and the cylinder (a). The objects (b2) and (c2) are due to multipath propagation. The object (d) is due to backside reflection of the cylinder surface. 
Using this data, scene analysis is performed where two basic types of objects are identified. The first type of object is a plane where the fact is used, that the direction vector is equal to the plane normal vector. Using a Hessian normal form, where the plane is described by a normal vector and the distance to the origin, planes with the same direction vector but different distances can easily be distinguished using a histogram of the distances. Distances with multiple points are classified as potential planes if their area spawns a defined threshold. Such data is excluded from further analysis.

Analysis of cylinders uses the fact that the cross product of any two direction vectors of the cylinder points into the axis of the cylinder. Using this fact, groups of vectors having their cross product pointing in the same direction, are obtained. This data is then projected on a plane with a normal vector identical to the axis of the cylinder, whereas reflection points on the cylinder surface appear as a circle. Within this image the center of the cylinder and its diameter are easily obtained.

The finally processed scene including the parameters of the object is shown in Fig. 4. The mirror objects (b2) and (c2) have been excluded for presentation purposes.

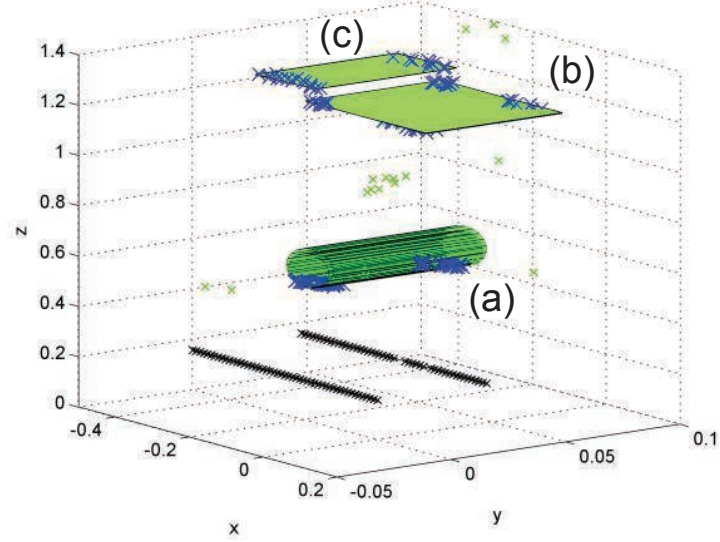

Fig. 4. Analyzed Scene showing the identified objects (a), (b) and (c). The door (b) has a plane normal of $(0.00,0.04,1.00)$ and a distance to the origin of $1.19 \mathrm{~m}$. The cabinet door (c) has an identical plane normal but a slightly larger distance of $1.21 \mathrm{~m}$. The cylinder radius was estimated with a diameter of $12 \mathrm{~cm}$ which is in reasonable agreement with the physical diameter of $10.5 \mathrm{~cm}$.

\section{Application "Recognition of Parameters of Objects transported on a Conveyor"}

Fig. 5 shows an example application where a group of two compact $3 \mathrm{D}$ sensors is used to analyze a moving object on a conveyor belt. At different positions of the object, at corresponding instants of time multiple reflection points of the object are obtained. The aim of this setup is to recognize the orientation and parameters of the object.

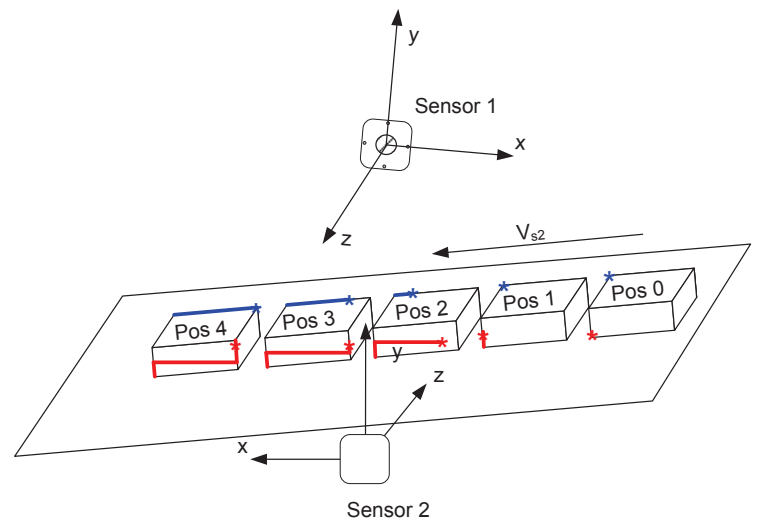

Fig. 5. At different time instants the box is observed at different positions by the array of sensors. The solid red line shows the trace of reflection points for sensor 2. Echoes at a specific point are marked with the symbol *. Similarly echoes for sensor 1 are shown in blue.

The following prerequisites are required for operation. Sensor 1 shall be placed such that it is above the top surface of the box; therefore echoes are solely due to edge and corner diffraction on the top edge. Sensor 2 shall be placed such that some echoes are on the front side of the box. Furthermore the direction of movement and the velocity of the belt need to be known. A suitable method for this is using an initial calibration device for measuring the direction and an incremental encoder during operation. In addition the array of sensors needs to be calibrated once, which is described in this section hereafter.

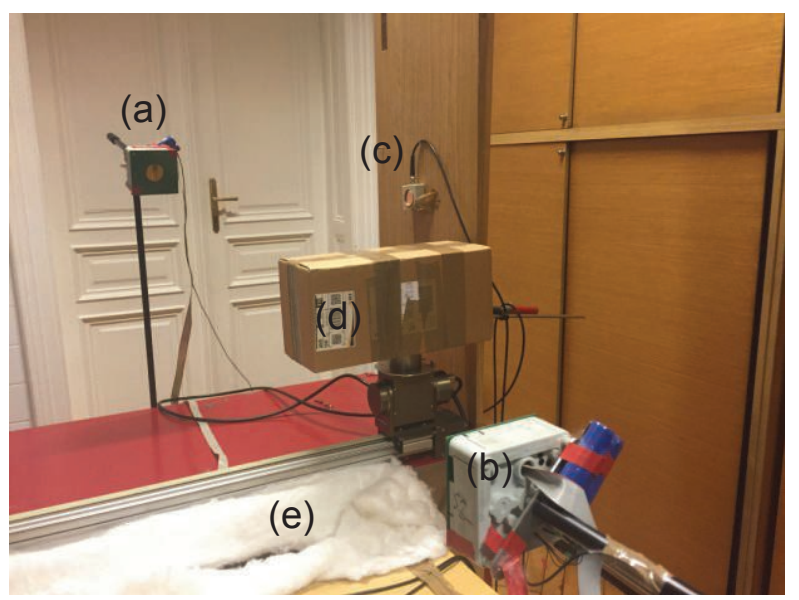

Fig. 6. Test setup showing two compact $3 D$ sensors where sensor 1 is labeled as (a), sensor 2 is labeled as (b). The object to recognize is a box (d) mounted on a linear belt (e). For initial calibration an active calibration device (c) can be used.

The physical test setup for the experiment is shown in Fig. 6. A small carton box with dimensions $38 \mathrm{~cm} \times 16 \mathrm{~cm} \times 18 \mathrm{~cm}$ has been 
mounted on a linear belt. Using the linear belt the box was moved $80 \mathrm{~cm}$ from the origin on the belt axis.

For a group of sensors to work together it is required to establish a common reference frame among them. Without loss of generality sensor 2 is used in this example. Using ToF both sensors can measure the 3D position of the respective other sensor in their local coordinate system. Furthermore each sensor can measure the position of the active calibration device in its local coordinate system. Let $R_{1 \rightarrow 2}$ be a rotation matrix and $S 1_{S 2}$ be the position of sensor 1 in the reference frame of sensor 2. Then a 3D vector $P_{S 1}$ in the reference frame of sensor 1 can be transformed into the reference frame of sensor 2 using (2).

$P_{S 2}=R_{1 \rightarrow 2} P_{S 1}+S 1_{S 2}$

$S 1_{S 2}$ is already known from the initial measurements. Using all four ToF measurements the rotational matrix can be obtained, as this problem can be rewritten such, that it is similar to Wahba's problem [8], often encountered in radar applications.

Having such a common reference coordinate system, measurements are taken at fixed time instants of time. As the box is moving along the axis of the belt echoes for sensor 2 first occur due to edge diffraction on the left front edge of the box. Afterwards echoes are due to reflection on the plane surface of the box. As the box moves out of the vicinity of the sensor 2 echoes are again due to edge diffraction on the right side. This can be seen in the principle drawing of Fig. 5 and the measurement in Fig. 6 . Echoes from sensor 1 are solely due to edge and corner diffraction. Fig. 7 shows the obtained measurement data for the test setup shown in Fig. 6. Classification of the box is performed as following. Using the data obtained by sensor 2 , echoes which have an identical direction vector are grouped. This data can be used to estimate the plane normal of the front side surface of the box. Using data from sensor 1 PCA is used. As variance of the data is mostly along the top edge of the box the largest eigenvector corresponds to the vector defined by the top edge. Furthermore, the extents of the box can be obtained from the PCA data using the smallest and largest values corresponding to the left and right corner of the box. From this data the length of the box can be directly obtained. As these points are also on the plane, which by the box assumption has the same normal vector as the front side of the box, the width of the box is obtained. The normal vector of the top surface can be estimated by the cross product of the plane vector and the edge. If, during initial calibration, a point on the surface of the belt is known the height can be estimated as well.

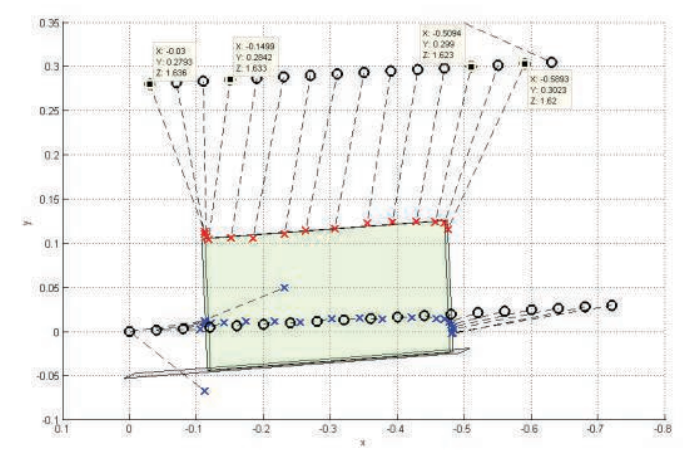

Fig. 7. Measurement showing $3 D$ reflection points of sensor 1 (red) and of sensor 2 (blue). The respective black circles are the (virtual) sensor positions as in reality the object moves and not the sensor. The dashed lines correspond to the direction vector of the echo.

A more robust implementation, not described herein, uses an improved PCA implementation. Estimation of the plane normal of the front side is already robust due to multiple data and grouping by the direction vectors. Estimation of the distances can be implemented using the median which gives a more robust estimate.

The estimated length of the box was $36,2 \mathrm{~cm}$ which is in reasonable correspondence with the physical length of $38 \mathrm{~cm}$. The width of the box was estimated by $16,05 \mathrm{~cm}$ which is in excellent agreement with the physical data. Orientation shows good correspondence as well but the reached accuracy was not verified up to now.

\section{Discussion of the Results}

The applications which are analyzed so far show the generic usability of the sensor and the algorithms for measurement data interpretation. Adaptions to the type of application have to be introduced into the system setup. In that case important aspects have to be considered and advantages or disadvantages of Ultrasonic measurements can be noticed:

- In industrial environments, mainly specular reflections and low signal damping take place, negative environmental impacts of Ultrasound can be disregarded

- Accuracy is based on a reference distance, Doppler effect can often be ignored as the used chirps have good Doppler resilience

- Automatic calibration of several sensors to get a common system of sensor coordinates can mostly be realized 
- Advantages of using Ultrasound: systems are cheap, work also in dark or foggy atmosphere

- Advantages of signal processing of Ultrasonic reflections: directly getting relevant information (edges, corners), direction towards the reflection point is perpendicular to the object surface (tangent plane in the reflection point)

- Disadvantages of using Ultrasound: limited number of measurement results because of specular reflection, strong air movements and thermal gradients are reducing accuracy

- Disadvantages of signal processing of Ultrasonic reflections: echo signals have a large dynamic range which has to be considered in system setup

- Measurement rate is depending on the object distances; in distance range of some meters measurements rates of 10 to 30 measurements per second are possible

\section{Conclusion}

Evaluating the performance of the compact 3D sensor in two example applications showed a promising wide applicability. Despite that both applications are tailored to a specific problem, the same and unmodified sensor has been used for both tasks. Due to its 3D localization capabilities, the fact that the direction of reflection corresponds locally to the surface normal, the low uncertainty, and high robustness reuse of such a sensor for different applications is possible. This is an important aspect as the sensor can be treated as a building block for more complex applications. Furthermore, any improvement on the sensor performance will immediately improve application performance. Current work focuses on extended parameter characterization capabilities with a static array of sensors for moving objects.

\section{References}

[1] L. Kleeman, R. Kuc, "Mobile Robot Sonar for Target Localization and Classification", The International Journal of Robotics Research, August 1995, doi: 10.1177/027836499501400401

[2] N. I. Giannoccaro, L. Spedicato, "Exploratory data analysis for robot perception of room environments by means of an in-air sonar scanner", Ultrasonics, August 2014, http://dx.doi.org/10.1016/j.ultras.2013.01.01 5

[3] A. Ochoa, J. Urena, A. Hernandez, M. Mazo, "Ultrasonic Multitransducer System for Classification and 3-D Location of Reflectors Based on PCA", IEEE Transactions on Instrumentation and Measurement, September 2009, doi: 10.1109/TIM.2009.2016820

[4] G. Kaniak, "Improved Ultrasonic Environment Perception in Air for Specular Scenes", PhD Thesis Vienna University of Technology, August 2010

[5] C. Walter, H. Schweinzer, "An accurate compact ultrasonic 3D sensor using broadband impulses requiring no initial calibration", 2012 IEEE International conference on Instrumentation and Measurement, May 2012, doi: 10.1109/I2MTC.2012.6229255

[6] C. Walter, H. Schweinzer, "Locating of Objects with Discontinuities, Boundaries and Intersections Using a Compact Ultrasonic 3D Sensor", 2014 IEEE International Conference on Indoor Positioning and Indoor Navigation, October 2014

[7] C. Walter, H. Schweinzer "Ultraschallsensor und Verfahren zur Ortung von Objekten", Austrian patent application, A 50415/2014

[8] G. Wahba, "A Least Squares Estimate of Spacecraft Attitude", SIAM Review, 1965 